\title{
The optimization of needle electrode number and placement for irreversible electroporation of hepatocellular carcinoma
}

\author{
Oyinlolu O. Adeyanju, Haitham M. Al-Angari, Alan V. Sahakian
}

Radiol Oncol 2012; 46(2): 126-135.

Received 16 December 2012

Accepted 7 March 2012

Correspondence to: Oyinlolu O. Adeyanju, PhD student with the Department of Biomedical Engineering, Northwestern University, 2145 Sheridan Rd., Evanston, IL 60208 USA. Phone: 847-467-2297; Fax: 847-491-4455; E-mail: lolu@northwestern.edu

Disclosure: No potential conflicts of interest were disclosed.

Background. Irreversible electroporation (IRE) is a novel ablation tool that uses brief high-voltage pulses to treat cancer. The efficacy of the therapy depends upon the distribution of the electric field, which in turn depends upon the configuration of electrodes used.

Methods. We sought to optimize the electrode configuration in terms of the distance between electrodes, the depth of electrode insertion, and the number of electrodes. We employed a 3D Finite Element Model and systematically varied the distance between the electrodes and the depth of electrode insertion, monitoring the lowest voltage sufficient to ablate the tumor, $\mathrm{V}_{\mathrm{IRE}}$. We also measured the amount of normal (non-cancerous) tissue ablated. Measurements were performed for two electrodes, three electrodes, and four electrodes. The optimal electrode configuration was determined to be the one with the lowest $\mathrm{V}_{\mathbb{R E}^{\prime}}$ as that minimized damage to normal tissue.

Results. The optimal electrode configuration to ablate a $2.5 \mathrm{~cm}$ spheroidal tumor used two electrodes with a distance of $2 \mathrm{~cm}$ between the electrodes and a depth of insertion of $1 \mathrm{~cm}$ below the halfway point in the spherical tumor, as measured from the bottom of the electrode. This produced a $V_{\text {IRE }}$ of $3700 \mathrm{~V}$. We found that it was generally best to have a small distance between the electrodes and for the center of the electrodes to be inserted at a depth equal to or deeper than the center of the tumor. We also found the distance between electrodes was far more important in influencing the outcome measures when compared with the depth of electrode insertion.

Conclusions. Overall, the distribution of electric field is highly dependent upon the electrode configuration, but the optimal configuration can be determined using numerical modeling. Our findings can help guide the clinical application of IRE as well as the selection of the best optimization algorithm to use in finding the optimal electrode configuration.

Key words: hepatocellular carcinoma; irreversible electroporation; optimization, electrode configuration

\section{Introduction}

Hepatocellular carcinoma (HCC), primary liver cancer, is a devastating cancer of the liver resulting in almost 700,000 deaths per year worldwide. ${ }^{1}$ HCC is generally caused by hepatitis B or C virus and is secondary to liver cirrhosis, which is most commonly caused by alcoholism and hepatitis $\mathrm{C}$ in the West. Hepatitis $\mathrm{C}$ infections are rising in western countries, leading to a rise in $\mathrm{HCC} .{ }^{2}$ The majority of patients who contract HCC die within a year. ${ }^{3}$ Although HCC is curable with surgical re- section, only $10-15 \%$ of patients can undergo surgical resection, and liver transplant waiting lists are prohibitively long. ${ }^{4}$

Many adjuvant therapies have been developed to treat liver tumors. Cryoablation works by freezing the tissue. Radiofrequency ablation (RFA) uses a high frequency (450-500 kHz) alternating current to oscillate cellular ions, inducing the generation of heat to treat the cancer, but RFA is limited in the amount of tissue that it can treat. RFA also suffers from a heat sink effect caused by the presence of blood vessels in the liver that convectively cool the 
tissue and thus reduce RFA's efficacy. ${ }^{5}$ Also, thermal ablation damages connective tissue and blood vessels. ${ }^{6}$

Irreversible electroporation (IRE) is a novel ablation method to treat HCC. ${ }^{7}$ IRE works by applying brief, high amplitude electric pulses to cancerous tissue. The electric field acts on the cellular membrane, raising the cell's transmembrane voltage, which can open semi-permanent to permanent aqueous pores in the membrane through which water soluble substances and ions can traverse the membrane. ${ }^{5}$ The permeabilization of the cell membrane disrupts the cell's homeostatic mechanisms and can result in the death of the cell. An advantage of IRE is that it leaves intact large blood vessels, nerves, ducts, etc. ${ }^{8}$

The effectiveness of IRE is highly dependent upon the distribution of the electric field in the tissue ${ }^{9,10}$, which in turn is dependent upon the configuration of electrodes and the amplitude of voltage applied. Miklavcic et al. demonstrated that tumor coverage with an adequate electric field is important for the effectiveness of the therapy. ${ }^{11}$ Various studies have looked into the effects of varying the electrode configuration for reversible electroporation applications. 5, 9, 10, 12-23 The majority of these were electrochemotherapy studies aiming to maximize the reversibly electroporated zones and to minimize the regions that were irreversibly electroporated. We are aware of only a few studies to date examining the effects of electrode configuration specifically for IRE therapy (Davalos et al. briefly reviewed some 2D simulated configurations in ${ }^{5}$ and Zupanic and Miklavcic looked at a treatment plan for IRE in ${ }^{24}$ and ${ }^{25}$ ), but the results for the reversible electroporation studies are useful in designing IRE treatments. Corovic et al. showed that the voltage, distance between electrodes, and the depth of electrode insertion were important parameters for the distribution of the electric field. ${ }^{18}$

There is a great deal of flexibility in terms of the configuration of electrodes. One could vary the number, shape, and size of electrodes, etc. as well as their placement. This study utilizes 3D finite element modeling studies to further develop knowledge in optimizing the needle electrode configuration for the purpose of treating liver cancer. We chose to study needle electrodes for their flexibility in placement and ability to treat both surface and deep-tissue tumors. That said, studies have shown that parallel plate electrodes may be more effective for surface tumors due to their ability to produce more uniform electric fields. ${ }^{26}$ Although the results are specific to $\mathrm{HCC}$, our hope is that the developed electrode configuration results would be useful for the ablation of other types of cancer and for motivating the use of different optimization algorithms.

\section{Materials and Methods}

\section{Finite Element Model}

We employed a 3D Finite Element Model using COMSOL ® 4.2 (COMSOL, Stockholm, Sweden) with MATLAB ${ }^{\mathrm{TM}}$ on a 64 bit $2.61 \mathrm{GHz}$ Dell Optiplex with an AMD ${ }^{\mathrm{TM}}$ 64X2 Dual Core Processor 5200+ with 3.93 GB of RAM running Microsoft Windows XP Professional Version 2003 Service Pack 2. We modeled the liver as a $3 \mathrm{D}$ rectangular object with dimensions $(18 \mathrm{~cm}$ width $\times 10 \mathrm{~cm}$ depth $\times 15 \mathrm{~cm}$ height), which was set to be the approximate dimensions of a human liver. The tumor was modeled as a sphere with a $2.5 \mathrm{~cm}$ diameter, which can be considered an average size for liver tumors ${ }^{27}$. The electrical potential was calculated using the Laplace equation for potential distribution:

$\nabla(\sigma \nabla \phi)=0$

$\sigma$ represents tissue conductivity and $\phi$ represents the electric potential. The electric field was calculated from the electric potential.

$$
E=-\nabla \phi
$$

E represents the electric field.

The boundaries of the cube were also set to be electrically insulating:

$$
-n \cdot J=0
$$

$\mathrm{n}$ represents the unit outward normal vector and $\mathrm{J}$ represents the current density. The tissue density was set to be $1050 \mathrm{~kg} / \mathrm{m}^{3},{ }^{28}$ and the electrode conductivity was set to be $4 \times 10^{6} \mathrm{~S} / \mathrm{m} .{ }^{29}$ The active electrode(s) were set to an electric potential $\phi=\phi_{0^{\prime}}$ and the electrode(s) that were not active were set to ground. A bounding box around the tumor was used in the FEM simulations to improve the quality of the meshing and computations. The mesh consisted of 29,505 elements.

The electrodes modeled in the study were platinum-iridium $(90 \% / 10 \%)$ electrodes represented by cylinders with $2.0 \mathrm{~cm}$ exposed length and 1 $\mathrm{mm}$ diameter with insulated portions above the exposed regions of the electrode. The normal liver was given a relative permittivity of $8.2 \times 10^{4}$, while the tumor relative permittivity was set to $9.9 \times 10.430$ We modeled the nonlinear change in the electrical conductivity due to the process of membrane permeabilization with a sigmoid relationship depending upon the electric field magnitude as according to Sel et al..$^{31}$ for the liver and Ivorra et al..$^{32}$ for the tumor. 


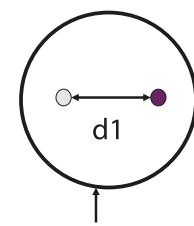

Tumor

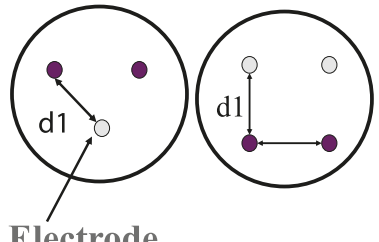

Electrode

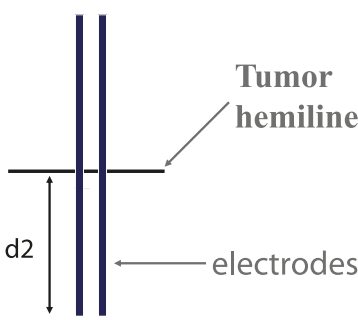

FIGURE 1. Depiction of the electrode configurations. (left) The purple circles represent active electrodes, and the lighter shaded electrodes are set to ground. The distance (d1) between electrodes and the depth of insertion (d2) was varied in the study.

The fit equation for the liver was:

$$
\sigma(E)=0.075+(0.27-0.075) *\left(\frac{1}{1+10 e^{\left(-1 / 3000^{*}(E-58000)\right)}}\right)
$$

The fit equation for the tumor was:

$$
\sigma(E)=0.166\left(2.154 * \exp \left(-e^{\left(-0.001234^{*}(E / 100 \mid-1500)\right)}\right)+1\right)
$$

E represents the electric field in $\mathrm{V} / \mathrm{m}$. Since the liver model from Sel et al. was determined from rabbit data, we changed the baseline value to 0.075 $\mathrm{S} / \mathrm{m}$ to coincide with human data derived from Haemmerich et al. 2009. ${ }^{33}$ The fit equation for the tumor was derived from mouse data, so the baseline conductivity was selected from Laufer et al. ${ }^{30}$ to be $0.166 \mathrm{~S} / \mathrm{m}$. The value from Laufer et al. was closer to the tumor fit equation's original baseline than the value from Haemmerich et al. for tumors, and conversely the chosen baseline value for the normal liver was closer to the original fit equation value from Sel et al. than that found in Laufer et al. It is well known that liver tumor conductivities tend to be higher than normal liver conductivities, and the ratio of $0.166 / 0.075 \approx 2.21$ is a reasonable ratio between the tumor and the liver. $18,28,34$

Mainly three different parameters of electrode configurations were analyzed for the study: 1) distance between electrodes 2) depth of electrode insertion 3) the number of electrodes. Basically, the parameter space for the distance between electrodes and the depth of electrode insertion was explored for three different electrode numbers: two, three, and four electrodes. The means of optimizing this was to increment the applied voltage at the active electrode(s) by $100 \mathrm{~V}$ intervals to the minimum value necessary to cover $95 \%$ of the tumor with an electric field sufficient to irreversibly electroporate the tumor tissue: $\mathrm{V}_{\text {IRE }}$. This electric field was set at $680 \mathrm{~V} / \mathrm{cm}$, as derived from Davalos et al.. ${ }^{5}$ The voltage was iterated, and the criterion for tumor ablation was determined via the MATLAB ${ }^{\mathrm{TM}}$ environment. Also, we were able to visualize the region of irreversible electroporation (tissue ablation) using an isosurface plotted in the $\mathrm{COMSOL}^{\circledR}$ 3D environment. $V_{\text {IRE }}$ was determined at several electrode distances and depths, and the results were tabulated for two, three, and four electrodes. The seven different distances between the electrodes that were measured were $1 \mathrm{~cm}, 1.5 \mathrm{~cm}, 2 \mathrm{~cm}, 2.5 \mathrm{~cm}, 3 \mathrm{~cm}$, $4 \mathrm{~cm}$, and $5 \mathrm{~cm}$. A distance of $1.9 \mathrm{~cm}$ was used for three electrodes as opposed to $2 \mathrm{~cm}$ to allow meshing with the electrodes close to the tumor border. The depths of insertion of the electrodes, as measured from the tumor's spherical hemiline to the bottom of the electrodes, were $0.5 \mathrm{~cm}, 1 \mathrm{~cm}$, $1.25 \mathrm{~cm}$, and $1.5 \mathrm{~cm}$ (Figure 1). In previous studies (data not shown), we tested shallower depths, but they had exorbitantly large $V_{\text {IREs }}$ and thus were not included for these experiments.

The applied pulse duration was selected to be $20 \mu \mathrm{s}$, which is at the lower end of the spectrum of pulse durations. ${ }^{35}$ This minimizes computational time and would also be useful for minimizing tissue resistive heating, which is directly related to pulse duration. A shorter pulse was not selected also because there should be an adequate amount of time to charge the membrane for IRE to be effective, and the membrane has a charge time of about $1 \mu \mathrm{s}{ }^{36}$ There has been no rigorous study as of yet examining the use of shorter versus longer pulses in the irreversible electroporation ablation of liver cancer cells as far as monitoring the size of the ablation zone. However, ultra-short pulses have been employed with very high electric fields (up to 300 $\mathrm{kV} / \mathrm{cm}$ ) to induce apoptosis by irreversibly electroporating inner organelles of the cell. ${ }^{37}$

The volume of normal (non-cancerous) tissue that was irreversibly electroporated-the Volume of Ablated Normal Tissue $\left(\mathrm{V}_{\mathrm{ANT}}\right)$ - was calculated in $\mathrm{COMSOL}^{\circledR}$ by integrating the volume of normal liver tissue in the simulated environment at or above $680 \mathrm{~V} / \mathrm{cm}$. $\mathrm{V}_{\text {IRE }}$ and $\mathrm{V}_{\mathrm{ANT}}$ were plotted versus distance at several depths, and some summary statistics were determined (mean, min, max).

\section{Results}

VIRE

The $\mathrm{V}_{\text {IRE }}$ results for two, three, and four electrodes are graphically depicted in Figure $2 \mathrm{~A}-\mathrm{C}$. The highest $V_{\text {IRE }}$ values consistently occur at the largest distances for all three sets of electrodes. The two electrode data show a parabolic minimum at a distance of $2 \mathrm{~cm}$. We can observe two regions from the results in Figure 2A, the first where the electrodes 

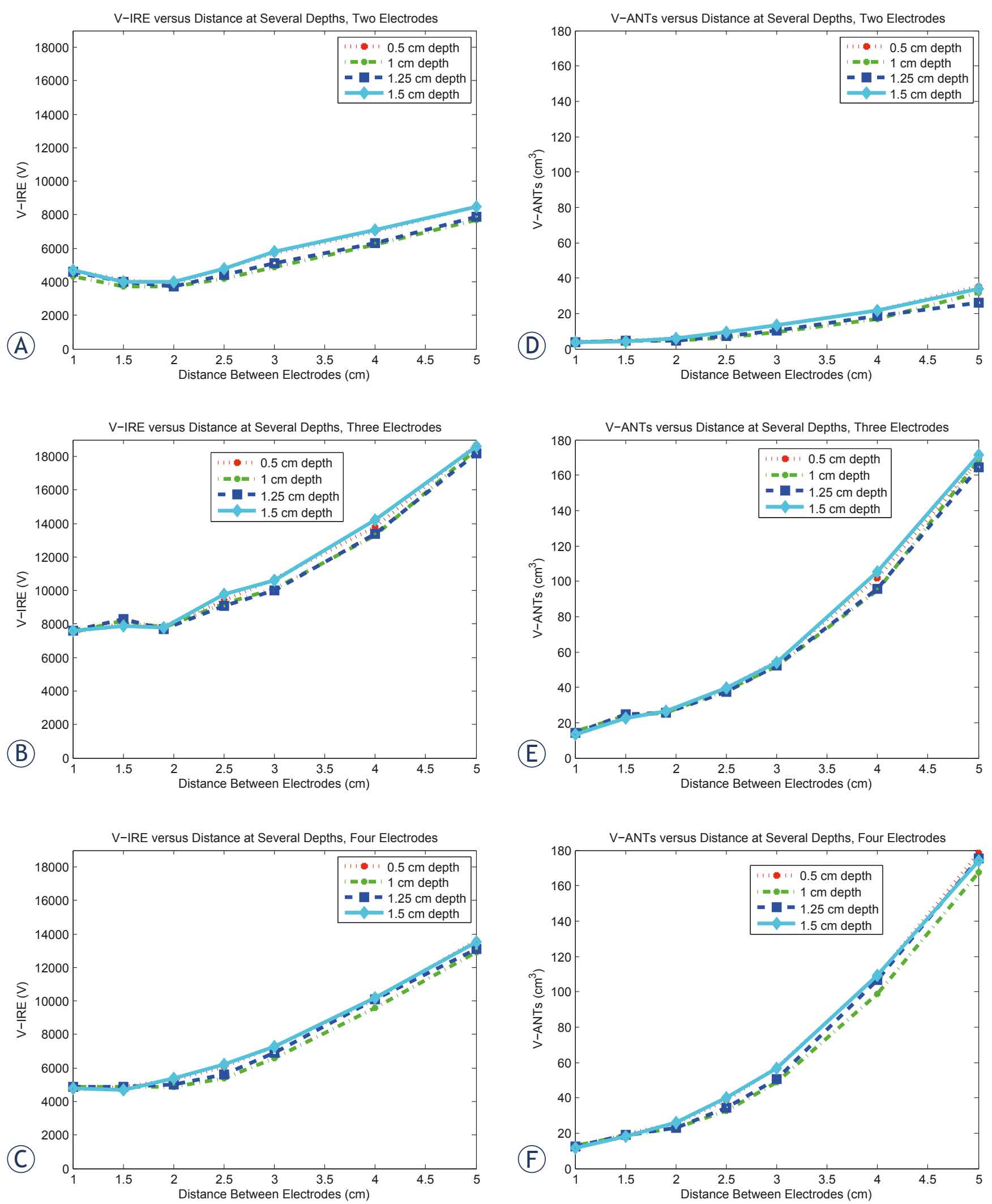

FIGURE 2 A-C. Graphical depiction of VIRE values upon distance at several depths. There appears to be a parabolic minima at $2 \mathrm{~cm}$ distance between the electrodes for the two electrode set, whereas the VIRE values for both three and four electrodes decrease linearly with the distance between the electrodes. D-F Graphical depiction of the dependence of the VANT values upon distance at several depths. The VANT values decrease with distance for all electrode setups. Two electrodes overall have the lowest VANT values, followed by four electrodes and then three electrodes. 
TABLE 1: Summary statistics from the three $\mathrm{V}_{\text {IRE }}$ tables $(\mathrm{V})$

\begin{tabular}{llll}
\hline Number of Electrodes & Minimum $\mathbf{V}_{\text {IRE }}$ & Mean $\mathbf{V}_{\text {IRE }}$ & Maximum V $_{\text {IRE }}$ \\
\hline TWo & 3700 & 5296 & 8500 \\
Three & 7500 & 10761 & 18700 \\
Four & 4700 & 7279 & 13600 \\
\hline \multicolumn{2}{l}{ summed $\mathbf{V}_{\text {IRE }}$ standard deviations while varying either distance or depth (V) } \\
\hline \multicolumn{2}{l}{ Parameter varying } & Two electrodes & Three electrodes \\
Distance & 6343 & 16036 & Four electrodes \\
Depth & 2133 & 1616 & 12873 \\
\hline
\end{tabular}

TABLE 2: Summary statistics from the three $\mathrm{V}_{\text {ANT }}$ tables $\left(\mathrm{cm}^{3}\right)$

\begin{tabular}{llll}
\hline Electrode \# & Minimum & Mean & Maximum \\
Two & 3.53 & 12.1 & 35.1 \\
Three & 13.2 & 60.5 & 171.3 \\
Four & 11.5 & 60.7 & 178.6 \\
\hline \multirow{2}{*}{ summed $\mathrm{V}_{\text {ANT }}$ Standard Deviations while varying either Distance or Depth } & \\
\hline Parameter varying & Two electrodes & & Four electrodes \\
Distance & 41.2 & 221 & 236 \\
Depth & 11.4 & 12.5 & 20.0 \\
\hline
\end{tabular}

are placed inside the tumor and the second where the electrodes are outside the tumor. The two-electrode data show a drop in the voltage as the distance increases from $1 \mathrm{~cm}$, before it rises again near the tumor-tissue boarder at a distance of $2.5 \mathrm{~cm}$. This behavior is observed for all electrode depths. The lowest $\mathrm{V}_{\text {IRE }}$ values occur at depths of $1 \mathrm{~cm}$ and $1.25 \mathrm{~cm}$, which correspond to the center of the electrode overlapping with the center of the tumor. The higher $\mathrm{V}_{\text {IRE }}$ values generally occur at a depth of $1.5 \mathrm{~cm}$ and then $0.5 \mathrm{~cm}$, indicating the highest $\mathrm{V}_{\text {IRE }}$ values are at the shallowest depth of insertion, and the deepest insertions (lower overlap) produce lower $\mathrm{V}_{\text {IRE }}$ values.

The lowest $V_{\text {IRE }}$ values for three and four electrodes appear to occur at the shortest distances between the electrodes. However, the three-electrode configuration showed an increase in the voltage as the distance increased, before it dropped again near the tumor-tissue boarder. Again this observation is independent of the electrode depth.

For the four-electrode configuration, there was either no change in the voltage (with $1 \mathrm{~cm}$ and $1.25 \mathrm{~cm}$ depth), an increase (0.5 cm depth), or a decrease in the voltage (with $1.5 \mathrm{~cm}$ depth) as a function of distance. When the electrodes were placed outside of the tumor, there was a monotonic increase in the required voltage for ablation as a function of distance for all of the electrode configurations and with all choices of depth. Overall, for all the different electrode configurations, there appears to be a smaller dependence of the $V_{\text {IRE }}$ values upon the depth of electrode insertion as compared to the distance between electrodes.

To visualize the differing ablation zones for the different electrode configurations, we examined a cross section of the center of the tumor, demarcating regions that were greater than or equal to 680 $\mathrm{V} / \mathrm{cm}$ for two, three, and four electrodes at a distance of $2.5 \mathrm{~cm}$ and a depth of $1 \mathrm{~cm}$ and plotted the ablation zones in Figure 3. Due to the asymmetric ablation shape with three-electrode configuration we tried moving the center of the electrode array and checked whether this would lower $V_{\text {IRE }}$ and $\mathrm{V}_{\text {ANT }}$ (Figure 3D). In fact this, lowered $\mathrm{V}_{\text {IRE }}$ and $\mathrm{V}_{\text {ANT }}$ from $9200 \mathrm{~V}$ and $38.4 \mathrm{~cm}^{3}$ to $4600 \mathrm{~V}$ and $16.3 \mathrm{~cm}^{3}$ respectively when moving the center of the electrode array $1 \mathrm{~cm}$ to the right of the center of the tumor. 


\section{Summary statistics for $V_{\text {IRE }}$ and influence of parameters (distance, depth) on $\mathrm{V}_{\text {IRE }}$}

Overall, the $\mathrm{V}_{\text {IRE }}$ values were lowest for two electrodes, followed by four electrodes and then three electrodes. Table 1 below shows some summary statistics concerning the three $\mathrm{V}_{\text {IRE }}$ tables as well as information about the influence of the different parameters upon the $\mathrm{V}_{\text {IRE}}$. The minimum, mean, and maximum $V_{\text {IRE }}$ were lowest for two electrodes followed by four electrodes and then three electrodes. The summed standard deviations for varying the distance were consistently greater for two, three, and four electrodes as compared to the summed standard deviations while varying the depth.

\section{$\mathrm{V}_{\text {ANT }}$ results}

The $\mathrm{V}_{\text {ANT }}$ results for two three, and four electrodes are depicted in Figure 2 D-F. The $\mathrm{V}_{\text {ANT }}$ values increase monotonically with distance for all electrode configurations and show the same variation with depth of electrode insertion as with the $V_{\text {IRE }}$ values where the depths of $1 \mathrm{~cm}$ and $1.25 \mathrm{~cm}$ consistently have the lower $V_{\text {ANT }}$ values relative to depths of $0.5 \mathrm{~cm}$ and $1.5 \mathrm{~cm}$. The two electrode setups have the lowest $V_{\text {ANT }}$ values, followed by four electrodes and three electrodes. The summary statistics are shown for $V_{\text {ANT }}$ values in Table 2. As with the $V_{\text {IRE }}$ values, the summed standard deviations are greater, when varying distance as opposed to depth.

The best configuration for the a) two b) three and c) four electrode setups, as according the lowest $V_{\text {IRE }}$ are: a) a distance of $2 \mathrm{~cm}$ and a depth of $1 \mathrm{~cm}$ $\left.\left(\mathrm{V}_{\text {IRE }}=3700 \mathrm{~V}\right) \mathrm{b}\right)$ a distance of $1 \mathrm{~cm}$ and a depth of $1 \mathrm{~cm}\left(\mathrm{~V}_{\text {IRE }}=7500 \mathrm{~V}\right)$ and $\left.\mathrm{c}\right)$ a distance of $2 \mathrm{~cm}$ and a depth of $1 \mathrm{~cm}\left(\mathrm{~V}_{\text {IRE }}=4900 \mathrm{~V}\right)$. The overall, global best choice for all electrode configurations was: the two-electrode configuration with a distance of $2 \mathrm{~cm}$ and a depth of $1 \mathrm{~cm}$ with $\mathrm{V}_{\text {IRE }} 3700 \mathrm{~V}$.

\section{Discussion}

The overall goal of our study was to find the optimal distance, depth, and number of electrodes for a simulated irreversible electroporation therapy of a subcutaneous HCC tumor. We systematically varied the distance and depth for three different sets of electrodes (two, three, and four electrodes) and looked at the outcome measures $V_{\text {IRE }}$ and $V_{\text {ANT }}$. The currently used Angiodynamics ${ }^{\mathrm{TM}}$ system uses only two electrodes at a time, but our study helps explore the potential benefits of different electrode
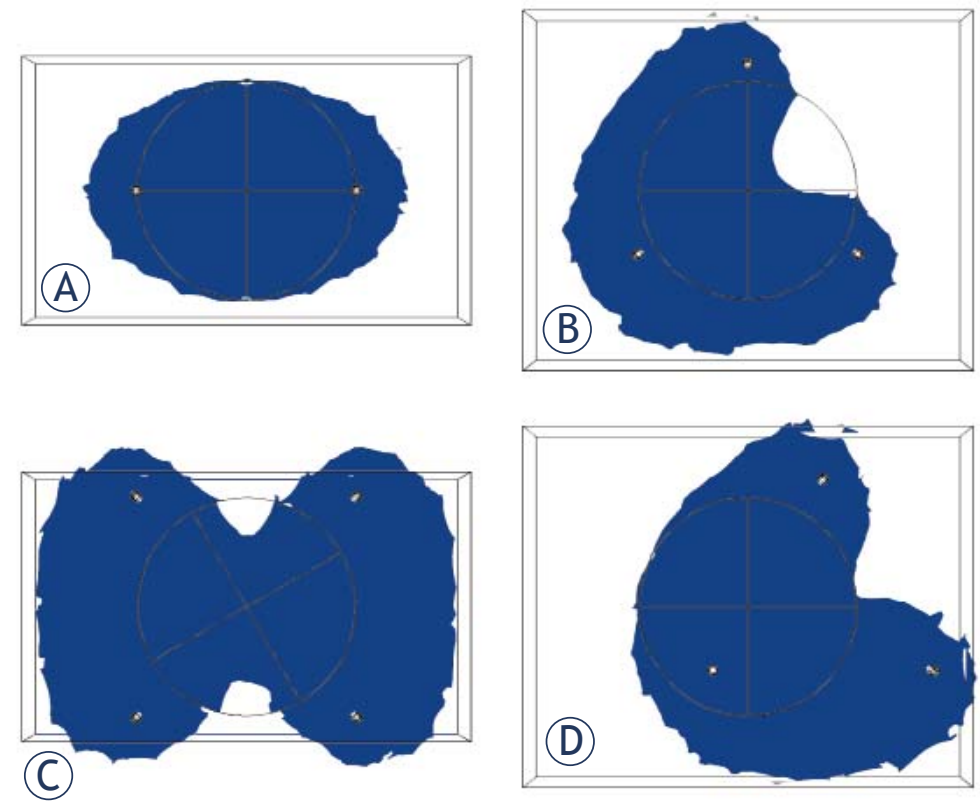

FIGURE 3. Cross section of the tumor (circle) and its ablation zone (blue) at an applied voltage of $5.05 \mathrm{kV}$, which corresponds to the VIRE for two electrodes at a distance of $2.5 \mathrm{~cm}$ and depth of $1 \mathrm{~cm}$ for A) two electrodes B) three electrodes C) four electrodes D) three electrodes with the center of the electrodes shifted to the right $1 \mathrm{~cm}$ Note the asymmetry in B and the narrowing of the ablation zone in $\mathrm{C}$. A bounding box around the tumor was used in the FEM simulations to improve the quality of the meshing and computations.

configuration patterns, particularly with the three electrode configurations. ${ }^{35}$ Our study builds upon findings from Zupanic et al. ${ }^{16}$ in that we include the possibility of electrodes within the tumor in our 3D simulations, which is advantageous, because the electric fields are highest near the electrodes and rapidly drop off with distance (i.e., the greatest amount of therapy occurs near the electrodes). It must be considered, however, that for some tumor cases it may not be possible to have the electrodes within the tumor. ${ }^{22}$

In looking at $\mathrm{V}_{\text {IRE' }}$, two electrodes produced the lowest $\mathrm{V}_{\text {IRE }}$, at a value of $3700 \mathrm{~V}$. It was unexpected that the two-electrode configuration produced the lowest outcome measure values $\left(\mathrm{V}_{\text {IRE }}\right.$ and $\left.\mathrm{V}_{\mathrm{ANT}}\right)$ whereas the three electrode configurations produced the highest, and the four electrode configuration outcome measures were in the middle. We expected that the greater surface area provided by a higher number of electrodes would cause the four-electrode configuration to have the lowest $\mathrm{V}_{\text {IRE }}$ values. This observation could be explained by a number of factors that played a role in the simulated tumor ablation process: a) the number of electrodes clearly plays a role as exhibited in Figure $3 \mathrm{~B}$ ) the high threshold of $95 \%$ tumor abla- 
tion c) the symmetry of electrode positioning and d) the distribution of the electric fields between the electrodes that occurred in the study.

The number of electrodes alters the shape of the ablated zone, as exhibited in Figure 3 where we visualize the difference in the ablation zone for two, three, and four electrode setups at a distance of $2.5 \mathrm{~cm}$ and depth of $1 \mathrm{~cm}$ with the same voltage of $5050 \mathrm{~V}$. For example, the two-electrode configuration in Figure $3 \mathrm{~A}$ has an elliptic ablation shape while the three-electrode has the most asymmetric shape. Another factor to consider is the different rise in the conductivity of the tissue and tumor due to the different nonlinear response to the rise in the electric field. As the electrodes are place outside and away from the tumor, the ablation shape has less effect as the whole tumor becomes more encompassed by the overall ablated zone, while the applied pulse has less effect on raising the tumor conductivity and more effect on raising the tissue conductivity.

The three-electrode configuration was the most asymmetric of the ones studied (relative to the tumor), as we used a symmetrical spherical tumor. Thus, it is reasonable to deduce that having an outcome whereby the tumor must be $95 \%$ ablated rather than $80 \%$ or $90 \%$ ablated can lead to using high voltages for configurations that use more than two electrodes. If one uses a threshold of $100 \%$ tumor ablation, the $\mathrm{V}_{\text {IRE }}$ values increase significantly. For example, the $\mathrm{V}_{\text {IRE }}$ becomes $4300 \mathrm{~V}$ instead of $3700 \mathrm{~V}$ for two electrodes with a distance of $2 \mathrm{~cm}$ and depth of $1 \mathrm{~cm}$, and $V_{\text {IRE }}$ becomes 16450 $\mathrm{V}$ instead of $4900 \mathrm{~V}$ for four electrodes with $2 \mathrm{~cm}$ distance and $1 \mathrm{~cm}$ depth. The asymmetric distribution of electrodes for three electrodes can more easily lead to incomplete tumor coverage (Figure 3B). This would explain why four electrodes, with greater symmetry of electrode patterning, would have lower outcome measures than that for three electrodes. The effect of moving the electrode center with respect to the tumor center in improving $\mathrm{V}_{\text {IRE }}$ and $\mathrm{V}_{\mathrm{ANT}}$ make us consider this feature when searching for the optimum electrode position, especially for configurations that result in asymmetric ablation zones. We noted the appearance of slightly higher $V_{\text {IRE }}$ values for three electrodes at $1.5 \mathrm{~cm}$ relative to the other nearby distances and reasoned that it may be caused by some effect due to both asymmetry and the nonlinear distribution of electric fields due to electrode positioning.

As for two electrodes versus four electrodes, we visualized the differing ablation zone shapes in Figure 3A,C. We saw that the four electrodes had a narrowing of the ablation zone near the center of the tumor, which would account for the higher $V_{\text {IRE }}$ values for the four electrodes as compared with the two electrodes. Also, the best choice for depth between 1 and $1.25 \mathrm{~cm}$ can be explained as more electrode surface area is facing the tumor with these depths. These findings suggest that clinicians applying IRE for subcutaneous tumors may find the use of two electrodes more beneficial. This information is also useful, because an increase in the number of electrodes can also lead to increased pain and discomfort for the patient and a greater risk of tumor seeding. ${ }^{38,39}$

The lowest $V_{\text {ANT }}$ values occur roughly at the same distances and depths as the lowest $\mathrm{V}_{\text {IRE }}$ values. The monotonic increase in the $V_{\text {ANT }}$ values as a function of distance is expected as more normal tissue volume experiences higher electric field intensities as the electrodes are placed further from the tumor. It is desirable in the application of IRE to minimize both the ablated normal tissue as well as the thermally ablated tissue, for the sake of preserving tissue structural elements (e.g., extracellular matrix, blood vessels, etc.). There appears to be a curvilinear relationship between the $V_{\text {ANT }}$ values and the distance between the electrodes, and thus clinicians should be careful to minimize the distance between the electrodes when applying the electric field across the tumor tissue to avoid the destruction of normal tissue.

We found that the distance between the electrodes held greater significance for determining what the optimal electrode configuration was, as compared with the depth of electrode insertion. This is apparent visually in Figure 2. Noting the greater influence of distance upon $V_{\text {IRE }}$ and the volume of normal and thermally ablated tissue could allow for more leeway in the electrode positioning as far as the depth of electrode insertion as compared to the distance between electrodes. It appeared that the dependence of the outcome measures with depth was greater for two and four electrodes as compared with three electrodes. Also, it seemed that the optimal depth was to have the center of the electrodes inserted close to or deeper than the center of the tumor. This finding corroborates what Zupanic et al. ${ }^{16}$ However, we build upon Zupanic et al. in that we include configurations where the electrodes were inserted into the tumor, and we simulated a greater tumor size (Zupanic et al. used sizes: $2 \mathrm{~mm}, 4 \mathrm{~mm}, 8 \mathrm{~mm}$ radii).

It would be interesting to conduct similar studies with different tumor sizes and to explore the effects of tumor size on the results. However, a tumor 
with a diameter of $2.5 \mathrm{~cm}$ is within the desired size range of $<3.0 \mathrm{~cm}$ preferred by clinicians who apply the therapy ${ }^{40}$, and it could be considered an average size. ${ }^{27}$ Also, it may not be practical to record results for every feasible tumor size. However, these findings and similar studies, besides serving as a reference for clinicians, has the value of better informing the use of dedicated optimization algorithms. Rather than having one sole output from an optimization algorithm, one gains a better understanding of how the fitness function $\left(\mathrm{V}_{\text {IRE }}\right)$ changes over the parameter space. In other words, the plots of $V_{\text {IRE }}$ over parameter space provide a better understanding of how the desired optimal value can vary with designated parameters. Thus, one can determine an optimization algorithm that best fits the fitness landscape, whether it is a gradient descent or genetic algorithm. Each algorithm has its strengths and weaknesses, and one could evaluate what the best optimization algorithm would be based upon these results. Also, by plotting the value of the objective function over the parameter space, one can know with greater certainty where the global minima versus local minima are. This is opposed to the use of an optimization method that would only output one or a limited number of values for the parameters, and one may not know if it is a local versus a global minima.

In the use of a dedicated optimization algorithm, it may be beneficial to relax the stringent standard of $95 \%$ ablation to $80-90 \%$ ablation to increase the diversity of potential solutions and utilize overlapping ablation zones. In clinical practice it may be better to attempt to ablate the tumor all at once to prevent future seeding. It would also be difficult to have overlapping IRE ablation zones due to the difficulty in visualizing the development of the ablation zone with high resolution in real time. Research in visualizing the ablation zone in real time has been done via electrical impedance tomography ${ }^{41}$, but significant advances must be made for this to become a reality. Some researchers have suggested that MRI may be a viable route for visualizing the ablation zone during IRE therapy. ${ }^{42-44}$

\section{Limitations}

The liver and tumor are assumed to be isotropic and homogenous. Future studies could also implement liver and tumor geometries from medical images. We do not account for the dependence of permeabilization on cell size, shape, and interaction with surroundings. ${ }^{31}$ Also, we did not study different electrode pulse sequence patterns or shapes, which can have a significant impact on the efficacy of the therapy. ${ }^{45}$ If electrode sequence activation patterns had been used, the four-electrode configurations could have been more efficacious, due to improved tumor coverage. ${ }^{46}$ Adjustments of the voltage to reach $\mathrm{V}_{\text {IRE }}$ were done in increments of 50 $\mathrm{V}$, and thus there is the possibility that some of the measurements were overestimated. Also, although we thought $\mathrm{V}_{\text {IRE }}$ to occur at $680 \mathrm{~V} / \mathrm{cm}$, it is possible that it occurs at a different cutoff value. However, the results would be expected to scale accordingly with a higher or lower threshold value for $V_{\text {IRE }}$.

The voltage amplitudes recorded from the study are higher than the maximum voltage supplied by NanoKnife $(3000 \mathrm{~V})^{35}$, but the information gleaned from the efficacy of different positions would still be useful to clinicians, and hopefully as technology improves, the observed voltages would become more feasible. Our optimum electrode position with two electrodes and a distance of $2.5 \mathrm{~cm}$ and $1 \mathrm{~cm}$ depth would ablate $82 \%$ of the tumor at the maximum system voltage of $3000 \mathrm{~V}$ (data not shown). It would also be useful to know thermal information about the electrode configurations used, but IRE pulse sequences use somewhere on the order of $90-100$ pulses $^{47}$, which would be impractical to examine for each electrode configuration with current computational capabilities.

\section{Conclusion}

We demonstrated that the optimal electrode configuration for applying IRE therapy for the ablation of HCC tumors could be determined using numerical modeling. We determined that it may be better to use two electrodes rather than three or four electrodes and that it is more important to be mindful of the distance between the electrodes rather than the depth of insertion of the electrodes to minimize the thermally ablated volume of tissue and the affected normal tissue. All of this information could serve as useful guidelines for physicians attempting to employ irreversible electroporation for the treatment of liver cancers. Although the model parameters we employed were specifically for liver cancer, the findings could be similar for other types of cancer e.g. kidney, breast, lung, etc. Our results corroborated previous findings that the distribution of the electric field in the tissue is highly dependent upon the electrode configuration, and future studies could further explore different electrode configurations with patient-generated tumor geometries and tissue properties. 


\section{Acknowledgements}

This work was supported by F31 CA150658 fellowship for PhDs from the National Cancer Institute of the NIH.

\section{References}

1. World health organization: Cancer. World Health Organization. 2012 Accessed: March 12, 2012. Available from: http://www.who.int/mediacentre/factsheets/fs297/en/index.html

2. Gomaa Al, Khan SA, Toledano MB, Waked I, Taylor-Robinson SD. Hepatocellular carcinoma: Epidemiology, risk factors and pathogenesis. World J Gastroenterol 2008; 14: 4300-8

3. Medicine Net: Liver cancer. Stoppler, M. 2011. Accessed: March 12, 2012. Available from: http://www.medicinenet.com/liver_cancer/article.htm

4. Forner A, Llovet JM, Bruix J. Hepatocellular carcinoma. Lancet 2012; Epub ahead of print.

5. Davalos RV, Mir IL, Rubinsky B. Tissue ablation with irreversible electroporation. Ann Biomed Eng 2005; 33: 223-31.

6. Daniels C, Rubinsky B. Electrical field and temperature model of nonthermal irreversible electroporation in heterogeneous tissues. J Biomech Eng 2009; 131: 071006.

7. Rubinsky B. Irreversible electroporation in medicine. Technol Cancer Res Treat 2007; 6: 255-60

8. Rubinsky B, Onik G, Mikus P. Irreversible electroporation: A new ablation modality--clinical implications. Technol Cancer Res Treat 2007; 6: 37-48.

9. Hofmann GA. Instrumentation and electrodes for in vivo electroporation. In. Jaroszeski MJ, Heller R, Gilbert R, editors. Electrochemotherapy, electrogenetherapy, and transdermal drug delivery. Electrically mediated delivery of molecules to cells. Totowa, New Jersey: Humana Press, 2000. p. 37-61.

10. Sel D, Mazeres S, Teissie J, Miklavcic D. Finite-element modeling of needle electrodes in tissue from the perspective of frequent model computation. IEEE Trans Biomed Eng 2003; 50: 1221-32.

11. Miklavcic D, Beravs K, Semrov D, Cemazar M, Demsar F, Sersa G. The importance of electric field distribution for effective in vivo electroporation of tissues. Biophys J 1998; 74: 2152-8.

12. Gilbert RA, Jaroszeski MJ, Heller R. Novel electrode designs for electrochemotherapy. Biochim Biophys Acta 1997; 1334: 9-14.

13. Sersa G, Cemazar M, Semrov D, Miklavcic D. Changing electrode orientation improves the efficacy of electrochemotherapy of solid tumors in mice. Bioelectrochem Bioenerg 1996; 39: 61-6.

14. Corovic S, Pavlin M, Miklavcic D. Analytical and numerical quantification and comparison of the local electric field in the tissue for different electrode configurations. Biomed Eng Online 2007; 6: 37.

15. Cindea NBF, Gournay F, Poignard C. Optimal placement of electrodes in an electroporation process. ESAIM: Proceedings 2010; 30: 34-43.

16. Zupanic A, Corovic S, Miklavcic D. Optimization of electrode position and electric pulse amplitude in electrochemotherapy. Radiol Oncol 2008; 42: 93-101.

17. Brandisky K, Daskalov I. Electrical field and current distributions in electrochemotherapy. Bioelectrochem Bioenerg 1999; 48: 201-8.

18. Corovic S, Zupanic A, Miklavcic D. Numerical modeling and optimization of electric field distribution in subcutaneous tumor treated with electrochemotherapy using needle electrodes. IEEE Transactions on Plasma Science 2008; 36: 1665-72

19. Miklavcic D, Semrov D, Mekid H, Mir LM. A validated model of in vivo electric field distribution in tissues for electrochemotherapy and for DNA electrotransfer for gene therapy. Biochim Biophys Acta 2000; 1523: 73-83.

20. Zupanic A, Corovic S, Miklavcic D, Pavlin M. Numerical optimization of gene electrotransfer into muscle tissue. Biomed Eng Online 2010; 9: 66.
21. Kos B, Zupanic A, Kotnik T, Snoj M, Sersa G, Miklavcic D. Robustness of treatment planning for electrochemotherapy of deep-seated tumors. J Membr Biol 2010; 236: 147-53.

22. Miklavcic D, Snoj M, Zupanic A, Kos B, Cemazar M, Kropivnik M, Bracko M, Pecnik T, Gadzijev E, Sersa G. Towards treatment planning and treatment of deep-seated solid tumors by electrochemotherapy. Biomed Eng Online 2010; 9: 10.

23. Sel D, Lebar AM, Miklavcic D. Feasibility of employing model-based optimization of pulse amplitude and electrode distance for effective tumor electropermeabilization. IEEE Trans Biomed Eng 2007; 54: 773-81.

24. Rubinsky B. Irreversible electroporation. Springer. Series in biomedical engineering. 2009. pp 203-217

25. Zupanic A, Miklavcic D. Method for treatment planning of tissue ablation by irreversible electroporation. In: Dössel O, Schlegel WC, eds. World congress on medical physics and biomedical engineering, september 7 - 12, 2009, Munich, Germany. Springer Berlin Heidelberg; 2009:150-153.

26. Edd JF, Horowitz L, Davalos RV, Mir LM, Rubinsky B. In vivo results of a new focal tissue ablation technique: Irreversible electroporation. IEEE Trans Biomed Eng 2006; 53: 1409-15.

27. Arrive L, Rosmorduc O, Dahan H, Fartoux L, Monnier-Cholley L, Lewin M, et al. Percutaneous acetic acid injection for hepatocellular carcinoma: Using ct fluoroscopy to evaluate distribution of acetic acid mixed with an iodinated contrast agent. AJR Am J Roentgenol. 2003; 180: 159-62.

28. Duck FA. Physical properties of tissue. San Diego: Academic Press Inc; 1990 p. 138

29. Elwassif MM, Kong $Q$, Vazquez M, Bikson M. Bio-heat transfer model of deep brain stimulation-induced temperature changes. J Neural Eng 2006; 3: 306-15.

30. Laufer S, Ivorra A, Reuter VE, Rubinsky B, Solomon SB. Electrical impedance characterization of normal and cancerous human hepatic tissue. Physiol Meas 2010; 31: 995-1009.

31. Sel D, Cukjati D, Batiuskaite D, Slivnik T, Mir LM, Miklavcic D. Sequential finite element model of tissue electropermeabilization. IEEE Trans Biomed Eng 2005; 52: 816-27.

32. Ivorra A, Al-Sakere B, Rubinsky B, Mir LM. In vivo electrical conductivity measurements during and after tumor electroporation: Conductivity changes reflect the treatment outcome. Phys Med Biol 2009; 54: 5949-63.

33. Haemmerich D, Schutt DJ, Wright AW, Webster JG, Mahvi DM. Electrical conductivity measurement of excised human metastatic liver tumours before and after thermal ablation. Physiol Meas 2009; 30: 459-66.

34. Deng ZS, Liu, J. Blood perfusion-based model for characterizing the temperature fluctuation in living tissues. Physica A: Statistical Mechanics and its Applications 2001; 300: 521-30.

35. Bertacchini C, Margotti PM, Bergamini E, Lodi A, Ronchetti M, Cadossi R. Design of an irreversible electroporation system for clinical use. Technol Cancer Res Treat 2007; 6: 313-20.

36. Edd JF, Davalos RV. Mathematical modeling of irreversible electroporation for treatment planning. Technol Cancer Res Treat 2007; 6: 275-86.

37. Gundersen M, Vernier PT, Marcu L, Li A, Zhu X, Gallam AZ, et al. Ultrashort pulse electroporation: Applications of high pulsed electric fields to induced caspase activation of human lymphocytes. Power Modulator Symposium 2002 and 2002 High-Voltage Workshop. Conference Record of the TwentyFifth International 2002

38. Cabibbo G, Craxi A. Needle track seeding following percutaneous procedures for hepatocellular carcinoma. World J Hepatol 2009; 1: 62-6.

39. Tezcan Y, Mehmet, K. Hepatocellular carcinoma with subcutaneous metastasis of the scalp. Radiol Oncol 2011; 45: 292-5.

40. Narayanan G. Irreversible electroporation for treatment of liver cancer. Gastroenterol Hepatol (N Y) 2011; 7: 313-6.

41. Granot $Y$, Ivorra A, Maor E, Rubinsky B. In vivo imaging of irreversible electroporation by means of electrical impedance tomography. Phys Med Biol 2009; 54: 4927-43.

42. Zhang Y, Guo Y, Ragin AB, Lewandowski RJ, Yang GY, Nijm GM, et al. M imaging to assess immediate response to irreversible electroporation for targeted ablation of liver tissues: Preclinical feasibility studies in a rodent model. Radiology 2010; 256: 424-32. 
43. Kranjc M, Bajd F, Sersa I, Miklavcic D. Magnetic resonance electrical impedance tomography for monitoring electric field distribution during tissue electroporation. IEEE Trans Med Imaging 2011; 30: 1771-8.

44. Hjouj M, Rubinsky B. Magnetic resonance imaging characteristics of nonthermal irreversible electroporation in vegetable tissue. J Membr Biol 2010; 236: 137-46.

45. Miklavcic D, Towhidi, L. Numerical study of the electroporation pulse shape effect on molecular uptake of biological cells. Radiol Oncol 2010; 44: 34-41.

46. Sahakian AV, Al-Angari HM, Adeyanju OO. Electrode activation sequencing employing conductivity changes in irreversible electroporation tissue ablation. IEEE Trans Biomed Eng 2012; 59: 604-7.

47. Zupanic A, Miklavcic, D. Tissue heating during tumor ablation with irreversible electroporation. Elektrotehniski Vestnik 2011; 78: $42-7$. 